\title{
Wavelet Analysis on the Temporal Series of Precipitation in Baoji
}

\author{
Yang Mi*, Xu Pan-pan, Qian Hui \\ College of Environmental Science and Engineering, Chang'an University, Xi'an, China \\ Emial address: \\ myang1993@163.com (Yang Mi), xupanpan0212@163.com (Xu Pan-pan), 55480981@qq.com (Qian Hui) \\ *Corresponding author
}

\section{To cite this article:}

Yang Mi, Xu Pan-pan, Qian Hui. Wavelet Analysis on the Temporal Series of Precipitation in Baoji. Science Discovery. Vol. 4, No. 3, 2016, pp. 189-196. doi: 10.11648/j.sd.20160403.16

Received: March 13, 2016; Accepted: April 15, 2016; Published: July 5, 2016

\begin{abstract}
Adopting the series data of precipitationfrom 1952 to 2013 in Baoji city, shaanxi province, China, using Morlet wavelet function, the seasonal variation of precipitation and the time series of the interannual variation of precipitation in Baoji nearly 60aare analyzed by wavelet analysis, reveals the multiple time scales change complex structure of Baoji precipitation, forecasts the precipitation change trend of four seasons in Baoji city. The results show that the seasonal and annual precipitationin Baoji has the characteristics of multi time scale, different scales show the different cycles, large scale periodic variations also include small scale periodic variations. As a whole, the performance for small scale changed seriously, no clear rules of special features, and there is obvious regularity of large scale. The time-frequency localization characteristic of wavelet analysis can show the fine structure of precipitation time series, and provide a new method for the analysis of the key water saving problems, such as multi time scale variation characteristics and short-term climate prediction.
\end{abstract}

Keywords: Baoji City, Wavelet Analysis, Precipitation

\section{基于小波分析的中国宝鸡降水时间序列的变化特征}

\author{
杨咪, 徐盼盼, 钱会 \\ 环境科学与工程学院, 长安大学, 西安, 中国
}

邮箱

myang1993@163.com（杨咪），xupanpan0212@163.com（徐盼盼），55480981@qq. com（钱会）

\begin{abstract}
摘要: 采用中国陕西省水利厅整编的1952 2013年宝鸡地区关于降水的系列资料, 采用Morlet小波函数, 对宝鸡近60a 来降水的季节变化和年际变化时间序列进行了小波分析，揭示了宝鸡市降水时间序列的变化特征，预测了宝鸡市四季 降水量以及年降水量的变化趋势。结果表明：宝鸡市的季节以及年降水都存在着多时间尺度的特征, 不同的时间尺度 表现为不同的循环交替, 大尺度的周期变化同时也包含着小尺度的周期变化。总体上大致表现为小尺度震荡剧烈, 没 有特别明显的规律特征, 而大尺度而存在着明显的规律。小波分析的时频局部化特性可展现降水时间序列的精细结构, 为分析气候多时间尺度变化特征及短期气候预测等节水关键问题研究提供了一种新方法。
\end{abstract}

关键词：宝鸡市，小波分析，降水量 


\section{1. 引言}

现在而言, 气候干旱, 蒸发量大, 降水稀少及其时空 分布不均匀是西北地区城市所面临的一个普遍问题。而中 国宝鸡市作为陕西省重要的重工业城市, 其处于渭河盆地 出山口的峡谷地带, 南部靠山北部靠塬, 渭河穿过其市中 心。

宝鸡市属于暖温带半湿润区, 季风盛行, 一年四季变 化比较分明, 冬夏季温差较大, 在季风环流和地形地貌的 影响下, 常出现严重的“伏旱”现象。多年平均气温 $12.9^{\circ} \mathrm{C}$, 极端最高气温 $41.6^{\circ} \mathrm{C}$, 极端最低气温 $-17^{\circ} \mathrm{C}$, 多年平均降 雨量 $679.1 \mathrm{~mm}$, 降雨一般多分布于7-10月份, 由西向东 逐渐增加, 中下游地区大于上游地区。随着城市的高速发 展, 工农业及生活用水量的迅速增加, 水资源供需不平衡 问题已经严重制约了其经济发展。同时, 由于降水所呈现 的阶段性变化特征, 所以十分需要对其变化特征加以分析, 从而尽可能的预测降水的变化趋势 $[1-2]$ 。

小波分析法, 是一种信号时间一尺度 (频率) 分析方法, 研究不同尺度周期随时间的变化情况, 被认为是傅立叶分 析方法的突破性进展。由于小波分析方法在时域与频域同 时具有良好的局部性, 并且可对信号进行时空多尺度分析, 可以聚焦到所研究对象的任意微小细节, 从而特别适合将 隐含在时间序列中各种随时间变化的周期振荡清楚地显 现出来 [3-4]。近几年在出降水量的时间序列中得到了广 泛的运用, 张愿章等 [5] 利用Morlet 小波分析对河南省 1951-2012年降水量进行分析, 张彦龙 [6]等的基于干旱指 数的宁夏干旱时空变化特征及其Morlet 小波分析, 李珠 [7] 等基于小波分析的无锡地区降水变化规律研究。

本文所用的资料为宝鸡地区1952-2013年以来近60a 的降水量资料。首先对四季 (冬季 $11-1$ 月, 春季 $2-4$ 月, 夏季 $5-7$ 月, 秋季 $8-10$ 月) 的年降水量进行线性拟合, 计 算出相应的线性倾向率, 然后采用的是Morlet小波函数进 行小波变换, 最后计算小波方差, 研究宝鸡地区降水变化 的时间结构及其周期变化规律。小波变换图能够映降水在 不同时间尺度上的周期震荡。信号的强弱通过小波系数的 大小来表示。等值线为正的用实线表示，代表降水偏多; 等值线为负的用虚线表示，代表降水偏少; 小波系数为零 则对应着突变点。小波方差图反映了能量随尺度年的分布, 可以确定一个时间序列中各种尺度扰动的相对强度。对应 峰值处的尺度称为该序列的主要时间尺度, 用以反映时间 序列的主要周期。

\section{2. 研究方法及其基本原理}

\section{1. 小波函数}

小波函数是指具有震荡特性、能够迅速衰减到零的一 类函数, 即:

$$
\int_{-\infty}^{+\infty} \varphi(t) d t=0
$$

小波函数有多种, 在小波分析中经常采用Marr小波、 Morlet小波、Wave小波、Mexican小波等。本文选用在时 域和频域局部性都较好的Morlet小波, 其函数为

$$
\varphi(\mathrm{t})=\mathrm{e}^{\mathrm{ict}} \mathrm{e}^{-\mathrm{t}^{2} / 2}
$$

式中, $c$ 为常数, $i$ 表示虚数。

Morlet小波是由一个周期函数经过Gaussian函数平 滑而得到的, 所以, 它的尺度因子 a 与Fourier变换中的周 期有着一一对应的关系: $T=\left[\frac{4 \pi}{c+\sqrt{2+c^{2}}}\right] \times a$ 。当取常数 $\mathrm{c}=6.2$ 时, $\mathrm{T}=1.00057 \mathrm{a} \approx \mathrm{a}$, 所以小波可以用来进行周期分 析。

\section{2. 小波变换}

小波变换是小波分析的核心。对于给定的、满足一定 条件的小波函数 $\varphi(\mathrm{t})$, 时间序列 $\mathrm{f}(\mathrm{t}) \in \mathrm{L}^{2}(\mathrm{R})$ 的连续小波变 换定义为:

$$
\mathrm{W}_{\mathrm{f}}(\mathrm{a}, \mathrm{b})=|\mathrm{a}|^{-\frac{1}{2}} \int_{-\infty}^{+\infty} \mathrm{f}(\mathrm{t}) \overline{\varphi(\mathrm{t})}\left(\frac{\mathrm{t}-\mathrm{b}}{\mathrm{a}}\right) d \mathrm{t}
$$

式中, $\mathrm{W}_{\mathrm{f}}(\mathrm{a}, \mathrm{b})$ 为小波变换系数; $\mathrm{a}$ 为尺度因子, 反 映小波的周期长度; $b$ 为时间因子，反映时间上的平移。

在实际应用中，水文时间序列大多是离散的。例如 $\mathrm{f}(\mathrm{k} \Delta \mathrm{t})(\mathrm{k}=1,2, \cdots, \mathrm{n} ; \Delta \mathrm{t}$ 为取样时间间隔), 则式 (2) 的离散形式为:

$$
\mathrm{W}_{\mathrm{f}}(\mathrm{a}, \mathrm{b})=|\mathrm{a}|^{-\frac{1}{2}} \Delta \mathrm{t} \sum_{\mathrm{k}=1}^{\mathrm{n}} \mathrm{f}(\mathrm{k} \Delta \mathrm{t}) \varphi\left(\frac{\mathrm{k} \Delta \mathrm{t}-\mathrm{b}}{\mathrm{a}}\right)
$$

$\mathrm{W}_{\mathrm{f}}(\mathrm{a}, \mathrm{b})$ 能同时反映时域参数 $\mathrm{b}$ 和频域参数 $\mathrm{a}$ 的特征, 它是时间序列 $f(t)$ 或 $f(k \Delta t)$ 通过单位脉冲响应的滤波器的 输出。根据 $\mathrm{W}_{\mathrm{f}}(\mathrm{a}, \mathrm{b})$ 随 $\mathrm{a}$ 和 $b$ 的变化, 可以绘出以 $b$ 为横坐 标, $\mathrm{a}$ 为纵坐标的关于 $\mathrm{W}_{\mathrm{f}}(\mathrm{a}, \mathrm{b})$ 的二维等值线图, 称为小 波变换系数图。通过分析小波变换系数图, 可以得到水文 时间序列在小波变换域中的小波变化特征, 从而揭示出水 文时间序列的多时间尺度演变特征和突变特征。

\section{3. 小波方差}

将时间域上关于 $\mathrm{a}$ 的所有小波变换系数的平方进行积 分, 即为小波方差。对于离散水文时间序列, 小波方差可 以采用下式计算:

$$
\operatorname{Var}(\mathrm{a})=\frac{1}{\mathrm{n}} \sum_{\mathrm{b}=1}^{\mathrm{n}}\left|\mathrm{W}_{\mathrm{f}}(\mathrm{a}, \mathrm{b})^{2}\right|
$$

式中, $n$ 为样本数; $\left|W_{f}(a, b)\right|^{2}$ 为小波变换系数 模平方。

小波方差随尺度 $\mathrm{a}$ 变化的过程, 称为小波方差图, 该 图能反映水文时间序列中所包含的各种尺度 (即周期) 的 波动及其强弱 (能量大小) 随尺度变化的特性, 图中各个峰 值分别对应着各个显著周期。因此, 通过小波方差图可以 很方便地确定一个时间序列中存在的主要时间尺度（即主 周期） $[8-10]$ 。 


\section{3. 实例分析}

\section{1. 春季降水量特征分析}

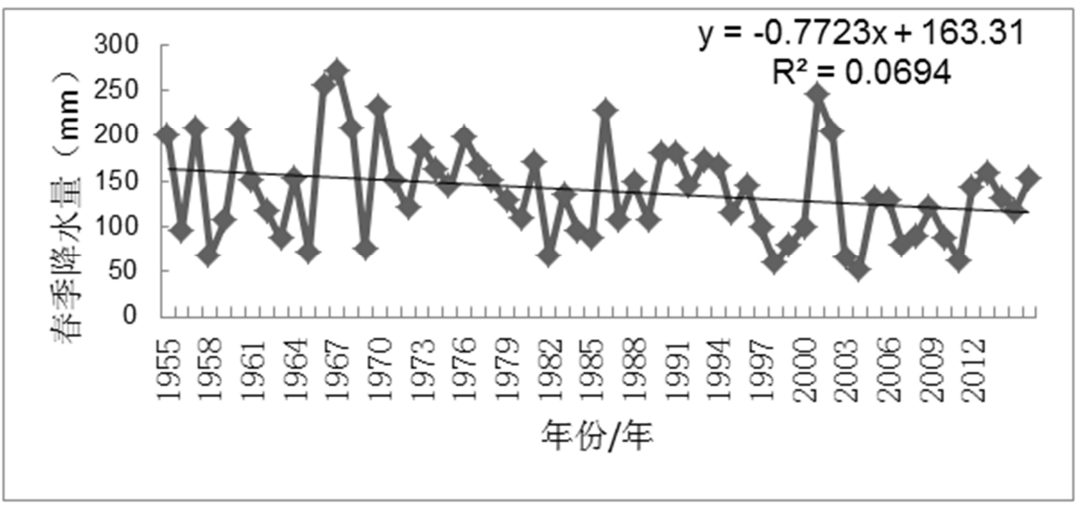

(a) 春季降水量变化趋势

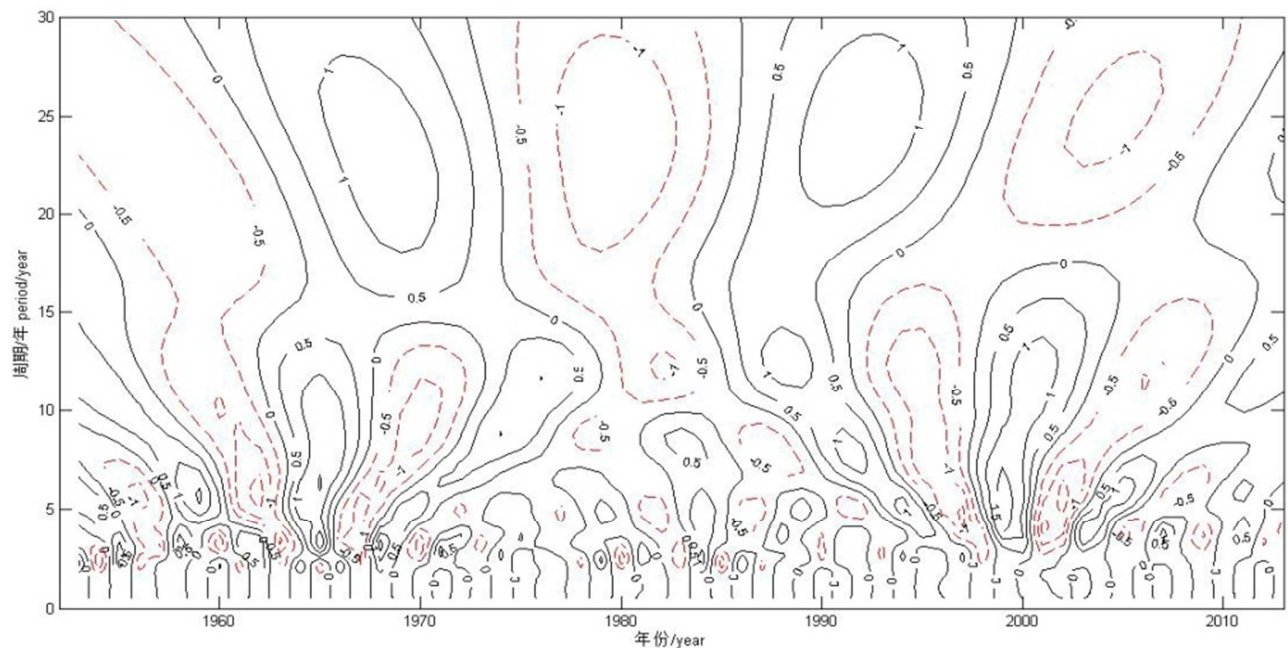

(b) 小波变换实部图

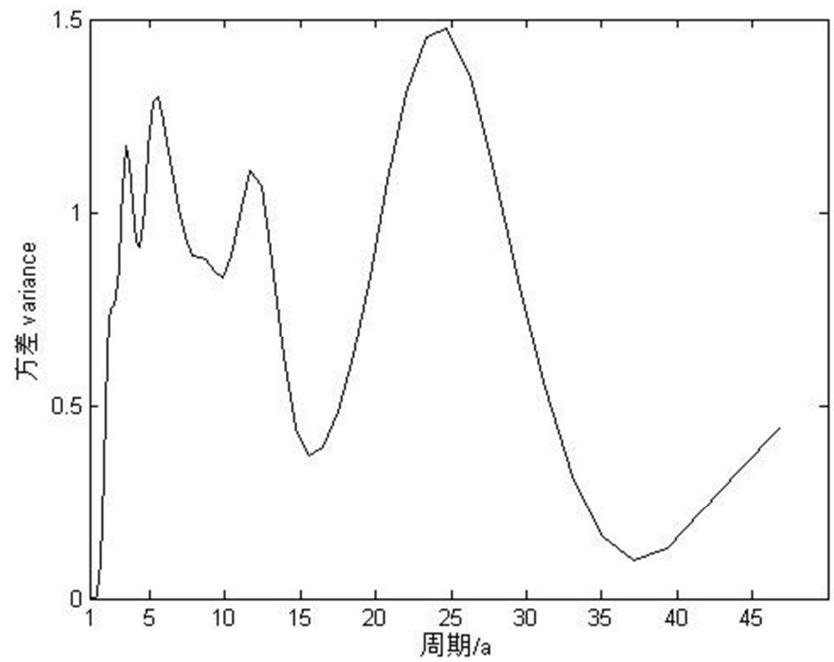

(c) 小波方差图

图1 春季降水量变化趋势 (a) 及其小波变换实部图 (b) 和小波方差图 (c)。 
由图1 $a$ 可以看出, 宝鸡市春季降水量 $(3 、 4 、 5$ 月降 水量总和) 总体呈下降趋势, 线性倾向率为 $-7.72 \mathrm{~mm} / 10 \mathrm{a}$ 。 由图 $1 b$ 可见，降水量在 $6 a$ 以下尺度下，周期表现的为剧烈 震荡, 并且没有明显的规律。随着时间尺度的增加, 在 6 - $15 \mathrm{a}$ 周期震荡则逐渐趋于平缓, 而且规律相对变得比较 清晰, 表现为多到少的5个循环交替, 且到2013年降水增 多的等值线并没有完全闭合，则说明降水逐渐增多的趋势

还是有可能持续。15a以上的尺度, 周期规律越来越清晰, 可以看出分别经历了由多到少 3 个循环。且从15a以上的大 尺度可以看出, 降水减少等值线的出现, 说明从大尺度上 降水减少的趋势并没有改变。从图 $1 \mathrm{c}$ 能看出, 在 $25 \mathrm{a}$ 周期 对应多到少 4.5 个循环交替, 宝鸡未来春季降水量很可能 会减少。

\section{2. 夏季降水量特征分析}

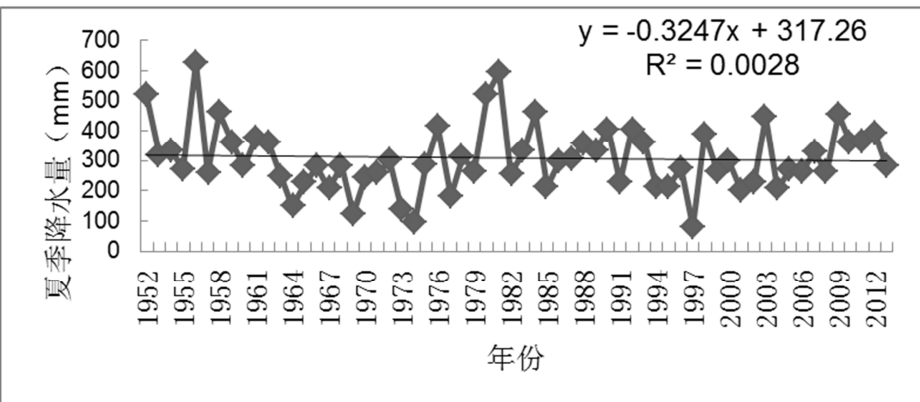

(a) 夏季降水量变化趋势

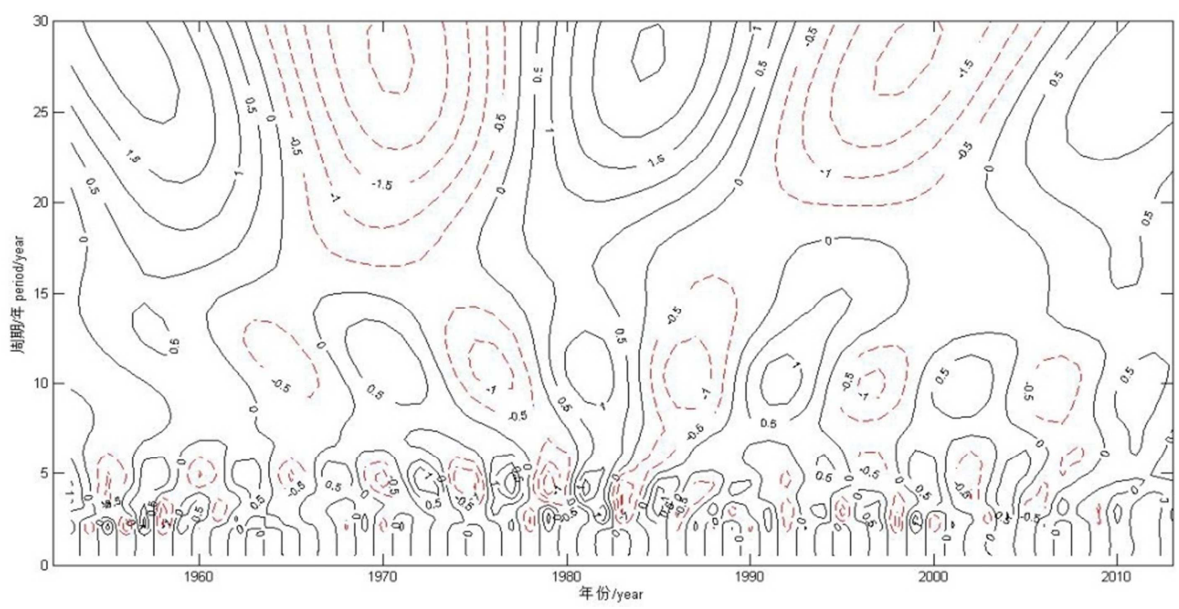

(b) 小波变换实部图

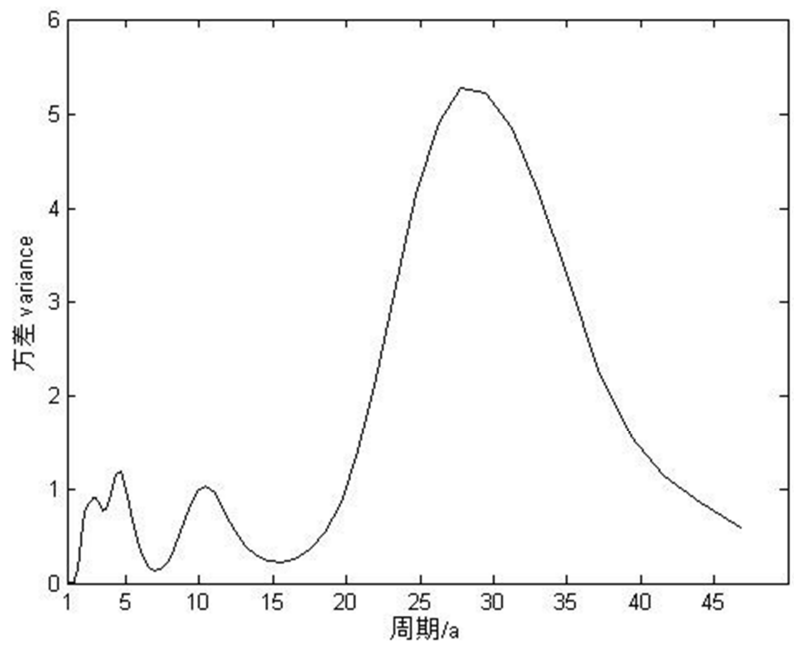

(c) 小波方差图

图2 夏季降水量变化趋势 (a) 及其小波变换实部图 (b) 和小波方差图 (c)。 
由图2a可以看出, 宝鸡市春季降水量（6、7、8月降 水量总和) 总体呈下降趋势, 线性倾向率为 $-3.24 \mathrm{~mm} / 10 \mathrm{a}$ 。 由图 $2 b$ 可见, 降水量在 $10 a$ 以下尺度下, 周期表现的为剧 烈震荡, 并且没有特别明显的规律。随着时间尺度的增加, 在 10-15a周期震荡则逐渐趋于平缓, 并且规律相对变得 比较清晰, 表现为多到少的 5 个循环交替, 且到2013年降

水增多的等值线并没有完全闭合，则说明降水逐渐增多的 趋势还是有可能持续。15a以上的尺度, 周期规律越来越 清晰, 可以看出分别经历了由多到少 2 个循环。说明未来 几年内宝鸡夏季降水量还会继续增多, 过后逐渐减少。从 图2c能看出, 在30a出现第一主周期, 规律性较为明显。

\section{3. 秋季降水量特征分析}

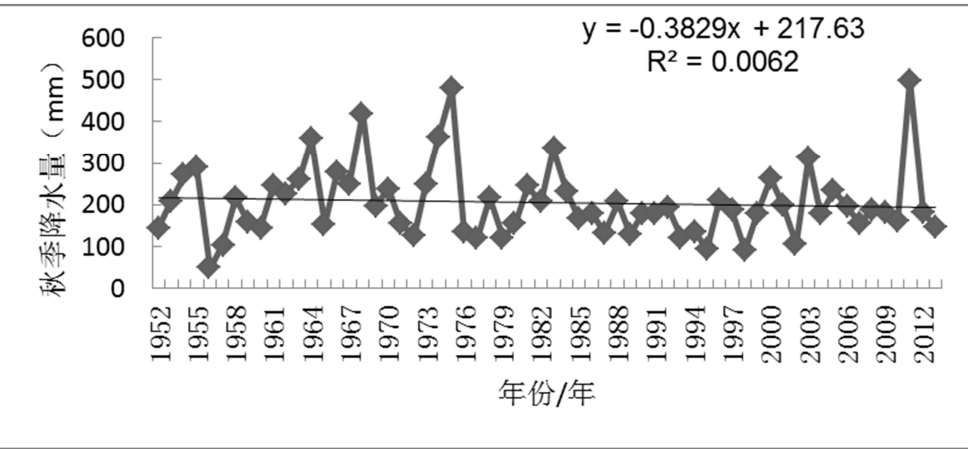

(a) 秋季降水量变化趋势

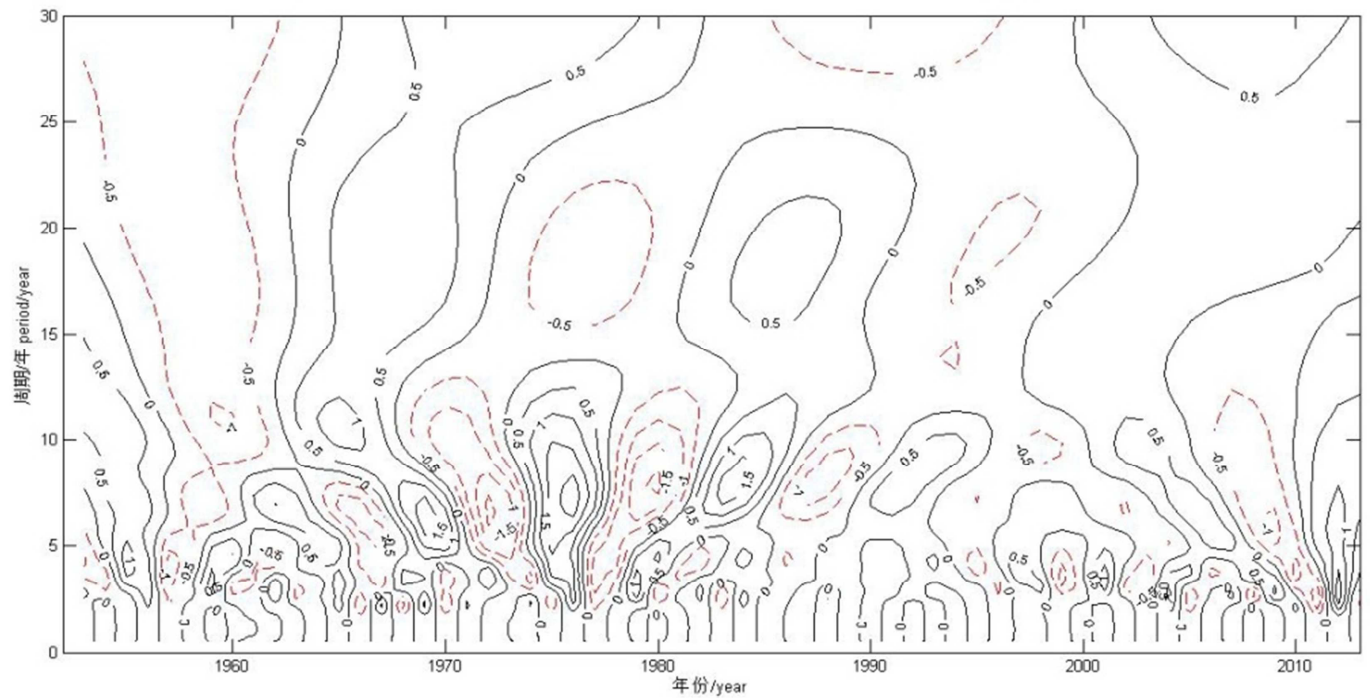

(b) 小波变换实部图

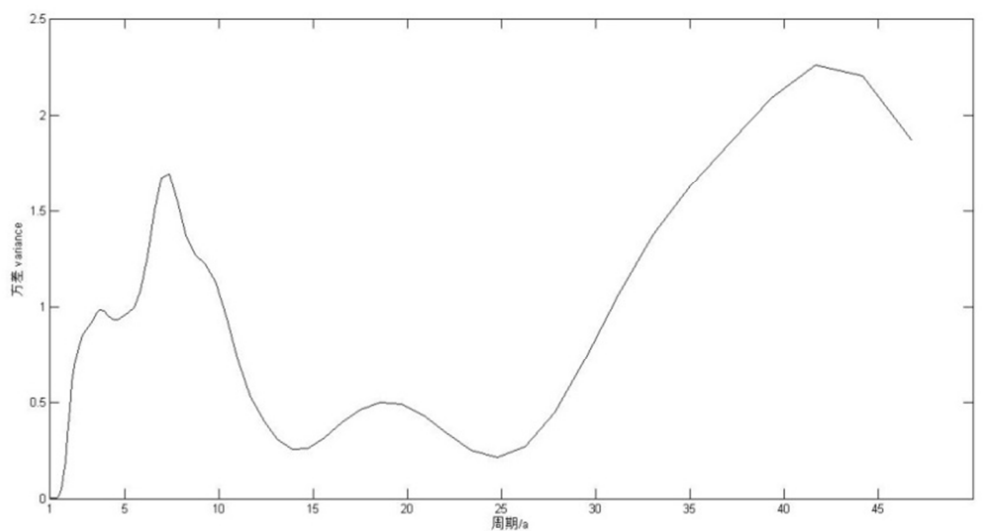

(c) 小波方差图

图3 秋季降水量变化趋势 (a) 及其小波变换实部图 (b) 和小波方差图 (c)。 
由图3a可以看出, 宝鸡市春季降水量（9、10、11月 降水量总和）总体呈下降趋势，线性倾向率为 $-3.82 \mathrm{~mm}$ / 10a。由图3b可见, 降水量在14a以下尺度下，周期表现 的为剧烈震荡, 并且没有明显的规律。而14a以上的尺度, 周期规律越来越清晰, 可以看出分别经历了由多到少 3 个 循环。且从14a以上的大尺度可以看出，降水增多等值线 的出现, 说明宝鸡未来降水仍可能持续增多, 过后再逐渐 减少。从图3c能看出, 在40a周期在大尺度上表现明显, 且不同的时间尺度突变点出现的位置有所不同。

\section{4. 冬季降水量特征分析}

(a) 冬季降水量变化趋势

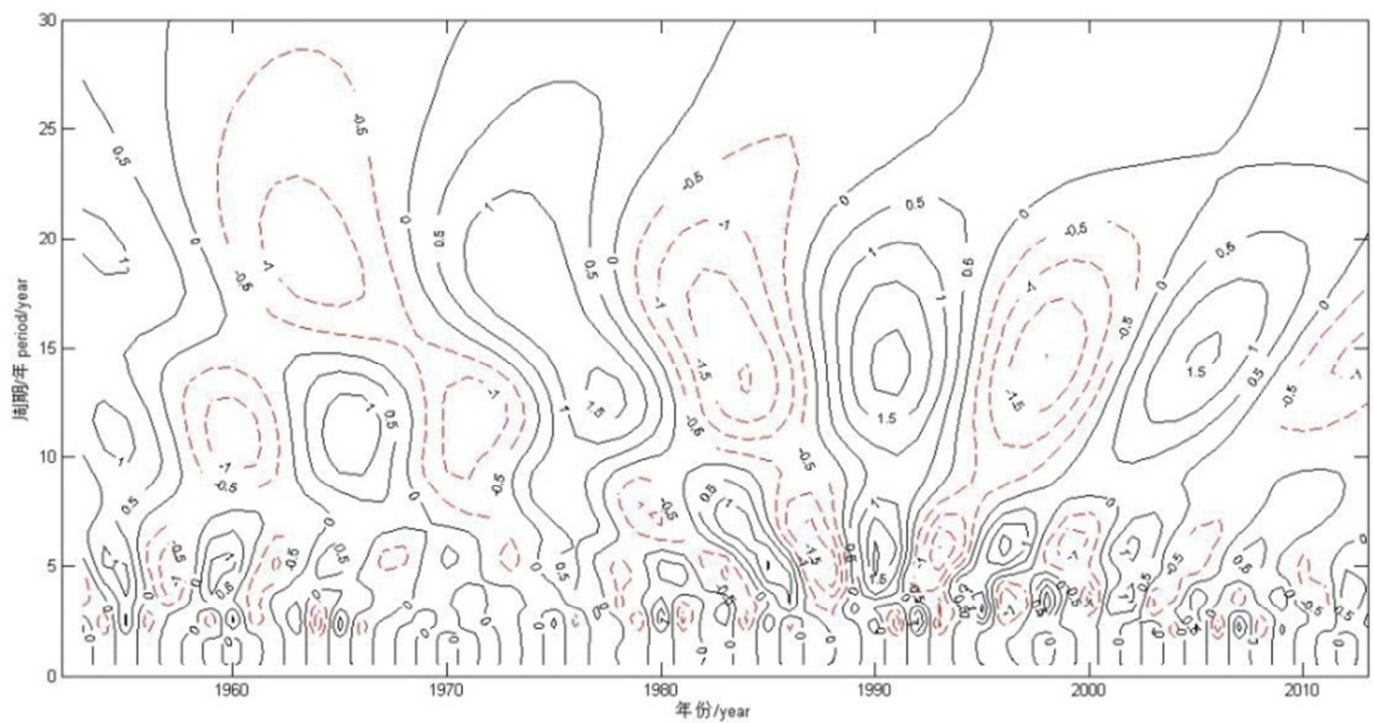

（b）小波变换实部图

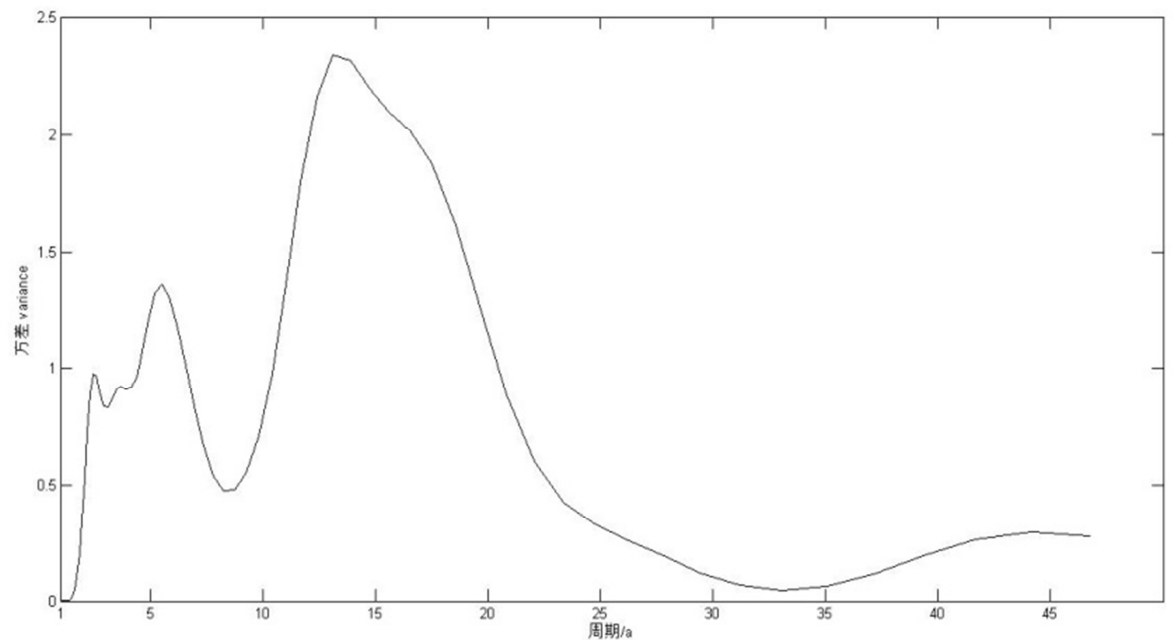

(c) 小波方差图

图4 冬季降水量变化趋势 (a) 及其小波变换实部图 (b) 和小波方差图 (c)。 
由图 $4 a$ 可以看出, 宝鸡市春季降水量 $(12 、 1 、 2$ 月降 水量总和) 总体呈上升趋势, 线性倾向率为 $0.15 \mathrm{~mm} / 10 \mathrm{a}$ 。 由图 $4 \mathrm{~b}$ 可见, 降水量在 $10 \mathrm{a}$ 以下尺度下, 周期表现的为剧 烈震荡, 并且没有明显的规律。随着时间尺度的增加, 10a
以上的尺度, 周期规律越来越清晰, 可以看出分别经历了 由多到少 4 个循环。从图 $4 \mathrm{c}$ 能看出, 在 $15 \mathrm{a}$ 周期对应多到少 3.5 个循环交替, 宝鸡未来春季降水量很可能会慢慢增加。

\section{5 . 年降水量特征分析}

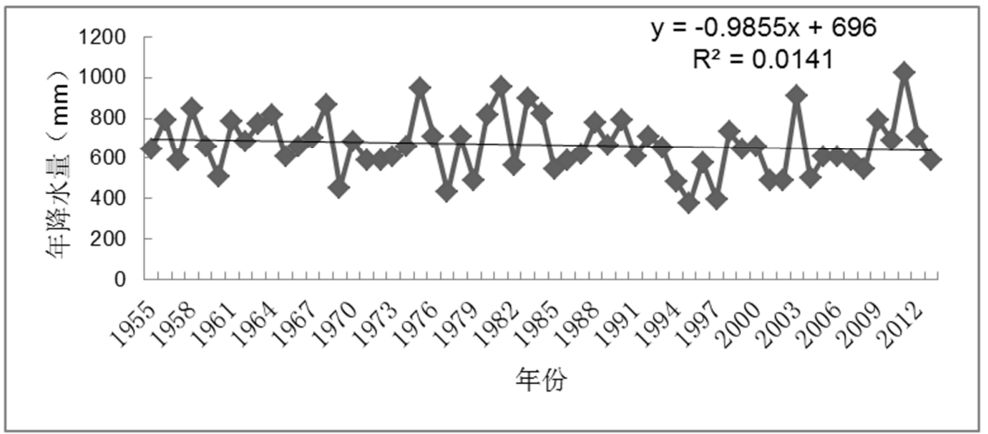

(a) 年降水量变化趋势

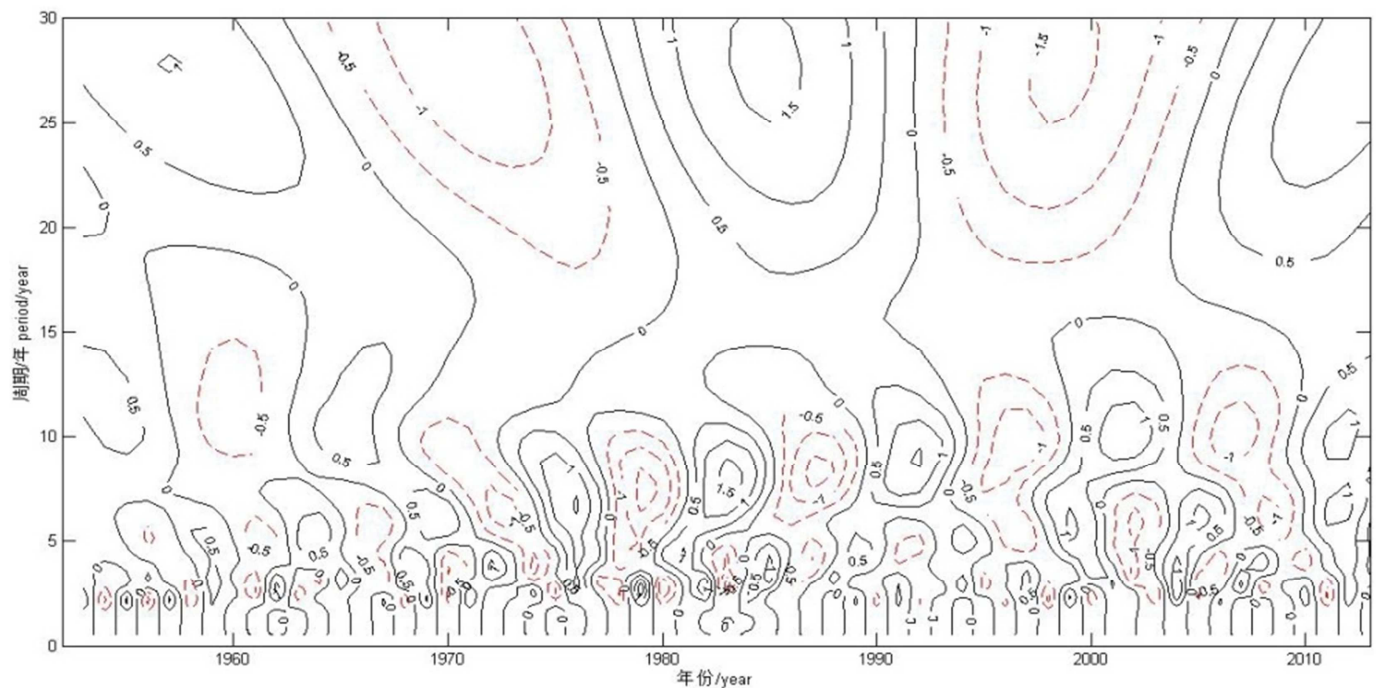

(b) 小波变换实部图

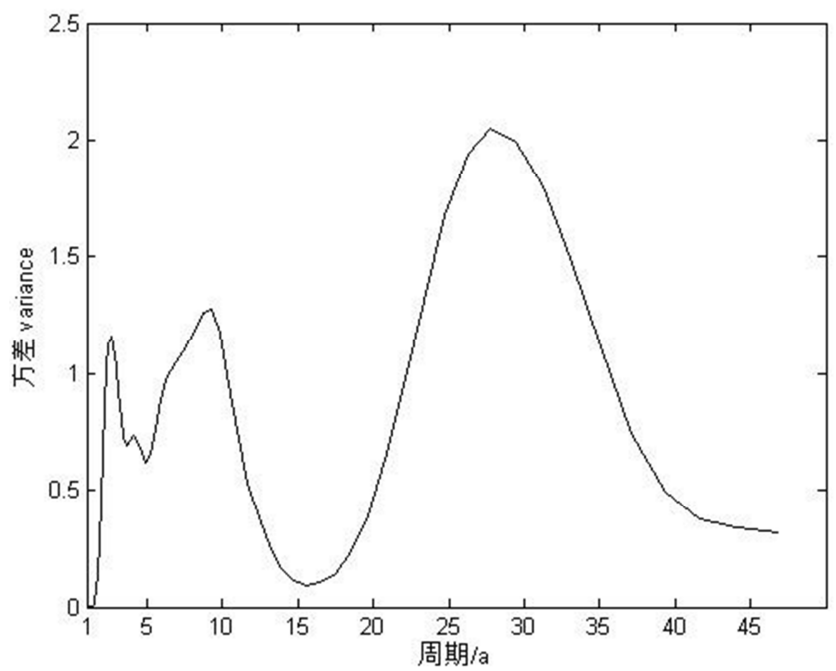

(c) 小波方差图

图5 年降水量变化趋势 (a) 及其小波变换实部图 (b) 和小波方差图 (c)。 
由图5a可以看出, 宝鸡市春季降水量（12、1、2月降 水量总和) 总体呈下降趋势, 线性倾向率为 $9.85 \mathrm{~mm} / 10 \mathrm{a}$ 。 由图5b可见，降水量在 $10 a$ 以下尺度下，周期表现的为剧 烈震荡, 并且没有明显的规律。随着时间尺度的增加, 在 10-17a周期震荡则逐渐趋于平缓, 并且规律相对变得比 较清晰, 表现为多到少的6个循环交替, 且到2013年降水 增多的等值线并没有完全闭合，则说明未来降水可能会逐 渐增加。 $17 \mathrm{a}$ 以上的尺度, 周期规律越来越清晰, 可以看 出分别经历了由多到少 2.5 个循环。从图 $4 \mathrm{c}$ 能看出, 在 $30 \mathrm{a}$ 周期对应多到少 3 个循环交替, 宝鸡未来春季降水量很可 能会慢慢增加。

\section{4. 结果与讨论}

（1）利用小波分析对中国宝鸡地区降水时间序列进 行分析, 发现了其在不同层次上的降水结构以及其变化规 律和突变, 同时预测了宝鸡市四季降水量以及年降水量可 能的变化趋势。

（2）中国宝鸡市的季节以及年降水都存在着多时间 尺度的特征，不同的时间尺度表现为不同的循环交替，大 尺度的周期变化也同时包含着小尺度的周期变化。而总体 上大致表现为在小尺度上震荡剧烈, 没有特别明显的规律 特征, 而大尺度而存在着明显的规律。

（3）通过本文可以看出，小波分析法是对降水的多 尺度分析及对未来降水的趋势进行预测的有效工具, 因此 利用小波分析对中国宝鸡地区的降水特征进行预测, 对今 后的工农业发展, 旱涝情况预测及水资源的合理利用提供 了合理的分析。

\section{致谢}

本文为水利部公益性行业科研专项经费项目 （201301084）的阶段性成果之一。

\section{参考文献}

[1] 唐春娥, 沈冰, 陈文让. 宝鸡市降水变化特征分析 $[J]$. 西 安建筑科技大学学报, 2007, 39(1):39.

［2］吕继强，莫淑红，沈冰。近半世纪宝鸡市干旱特征及模型 预测研究 [J]. 北京师范大学党报, 2010, 46(3)：333.

［3］邱海军，曹明明，曾涁. 基于小波分析的西安降水时间序 列的变化特征 $[J]$. 中国农业气象, 2011, 32(1) 1:23-27.

［4］孙然好, 潘保田, 牛最荣, 等. 河西走廊近年来地表水资 源时间序列的小波分析 [J]. 干旱区地理，2005，28(4)： 455-459.

［5］张愿章, 段永康, 郭春梅, 等. 河南省1951-2012年降水量 的Morlet小波分析 $[J]$. 人民黄河, 2015，37(10):25-28.

[6] 张彦龙, 刘普幸, 王允. 基于干旱指数的宁夏干旱时空变 化特征及其Morlet小波分析 [J]. 生态学杂志, 2015, 34 (8): 2373-2380.

［7］李珠, 沈浩, 顾春霞. 基于小波分析的无锡地区降水变化 规律研究 [J]. 安徽农业科学, 2015 (29) : 198-200.

[8］刘东，付强. 基于小波变换的三江平原低湿地井灌区年降 水序列变化趋势分析 $[J]$. 2008, 28 (3):380-381.

[9] 张耀存, 张录军. 东北气候和生态过渡区近 50 年来降水和 温度概率分布特征变化 $[\mathrm{J}]$ 。 地理科学, 2005 , $25(5): 561-566$.

[10] 王文圣, 丁晶, 李跃清. 水文小波分析 $[M]$. 北京：化学工 业出版社, 2005:115-141. 